\title{
Confined growth of CdSe quantum dots in colloidal mesoporous silica for multifunctional nanostructures
}

\author{
Chunguang $\mathrm{Li}^{1,2}$, Zhenda $\mathrm{Lu}^{2}$, Qiao Zhang ${ }^{2}$, Jianping $\mathrm{Ge}^{2}$, Shaul Aloni ${ }^{3}$, Zhan Shi ${ }^{1}$ and Yadong Yin ${ }^{2 *}$
}

\begin{abstract}
We report a new and convenient strategy for incorporating fluorescent semiconductor nanocrystals into silica hosts for the synthesis of multifunctional nanostructures. Mesoscale porosity was first created in conventional Stöber silica spheres by chemical etching under the protection of polymeric ligands. Uniform and highly luminescent CdSe nanocrystals were then directly grown in the porous silica network by reacting the silica spheres in a growth solution at high temperature. The confinement of silica network led to slower nanocrystal growth and subsequently smaller CdSe dots with blue shifted fluorescence compared with those without confinement. The loading number of CdSe nanocrystals can be easily tuned by changing the degree of porosity of the silica. The advantages of this strategy include simplicity as no special surface treatment processes are needed, general applicability to silica hosts of various shapes and sizes, high flexibility in tuning the dimensions of both the active nanocrystals and host particles, and ample opportunities for incorporating multiple functionalities. With the demonstration of a porous $\mathrm{Fe}_{3} \mathrm{O}_{4} @ \mathrm{SiO}_{2} / \mathrm{CdSe}$ composite structure with combined magnetic and optical properties, we believe this strategy may provide a platform for the fabrication of a large variety of multifunctional composite nanostructures.
\end{abstract}

\section{INTRODUCTION}

Colloidal semiconductor nanocrystals — so called quantum dots (QDs) - are widely used nanoscale fluorescent probes in biomedical applications due to their unique optical properties, such as size-dependent band gaps, extremely bright emission, a wide range of accessible colors, and remarkable photo-stability comparing to the organic dyes [1-7]. In order to enhance fluorescent response, decrease toxicity, and enable multi-functionalities, the quantum dots are frequently incorporated into a host matrix such as polymer or silica microspheres [7-18]. Among all the host materials, silica has been intensively investigated and broadly utilized, which is believed to benefit from its low reactivity, colloidal stability, biological compatibility and easy surface modification. Conventionally, semiconduct- ing QDs are synthesized first and then incorporated into silica microspheres through impregnation or other methods [19-26]. Often the silica microspheres are very large (several micrometers), which may increase the loading of QDs to some extent, but on the other hand may limit their application in biomedical field.

Loading nanoparticles to silica microspheres is of general interest to many fields as it allows the production of colloidal composites with multiple functions. Here, we introduce a new and reliable process for creating porosity in silica spheres and then loading them with functional nanoparticles, by using CdSe QDs as an example. In conventional methods, porosity is created in silica spheres during their synthesis by using surfactants as templates (porogens), which makes it difficult to extend to silica spheres of smaller dimensions. In our approach, silica spheres are synthesized using regular Stöber process which allows large variation in size, and then etched to produce desired porosity. Instead of incorporating the pre-made QDs into host silica spheres, our process directly grows CdSe QDs inside the silica pores, producing composite spheres with relatively high loading of QDs. We also show that the growth of QDs is limited by the confinement of the pores so that they emit at shorter wavelength than those without confinement. The advantage of our process is that other functional materials can be easily incorporated in the silica spheres before loading of QDs, making it easier to create multifunctional materials. This feature was demonstrated by synthesizing $\mathrm{Fe}_{3} \mathrm{O}_{4} @ \mathrm{SiO}_{2} / \mathrm{CdSe}$ composite structures that display both fluorescence and magnetic responses.

\section{EXPERIMENTAL}

\section{Chemicals}

Tetraethyl orthosilicate (TEOS, 98\%), sodium hydroxide (98.8\%), ammonium hydroxide aqueous solution (28\%), and isopropanol (denatured) were purchased from Fisher

\footnotetext{
${ }^{1}$ State Key Laboratory of Inorganic Synthesis \& Preparative Chemistry, Jilin University, Changchun 130012, China

${ }^{2}$ Department of Chemistry, University of California, Riverside, CA 92521, USA

${ }^{3}$ The Molecular Foundry, Lawrence Berkeley National Laboratory, Berkeley, CA 94720, USA

*Corresponding author (email: yadong.yin@ucr.edu)
} 
Scientific. Poly(vinylpyrrolidone) (PVP, $\left.M_{\mathrm{w}}=10,000\right)$, trioctylphosphine oxide $\left(\left(\mathrm{C}_{8} \mathrm{H}_{17}\right)_{3} \mathrm{P}=\mathrm{O}\right.$ or TOPO, $\left.99 \%\right)$, trioctylphosphine $\left(\left(\mathrm{C}_{8} \mathrm{H}_{17}\right)_{3} \mathrm{P}\right.$ or TOP, $\left.97 \%\right)$, oleylamine $\left(\mathrm{C}_{18} \mathrm{H}_{37} \mathrm{~N}\right.$, Tech., 70\%), stearic acid $\left(\mathrm{C}_{18} \mathrm{H}_{36} \mathrm{O}_{2}, 99 \%\right)$, selenium (Se, 99.99\%), and cadmium oxide (CdO, 99.5\%) were obtained from Sigma-Aldrich. All chemicals were used as received without further purification.

\section{Synthesis of porous silica spheres}

Silica spheres were prepared through the well-known Stöber method [27]. In a typical process for producing samples with an average diameter of $450 \mathrm{~nm}, 100 \mathrm{~mL}$ isopropanol was first mixed with $20 \mathrm{~mL}$ ammonium hydroxide aqueous solution and $5 \mathrm{~mL}$ distilled water under vigorous stirring. After $\sim 2 \mathrm{~min}, 5 \mathrm{~mL}$ of TEOS was added to the mixture which was kept under stirring for another $2 \mathrm{~h}$ at room temperature. The silica spheres were collected by centrifugation and washed with water for three times, and then etched by $\mathrm{NaOH}$ solution under protection of PVP $[28,29]$. Typically, the silica particles were re-dispersed in $60 \mathrm{~mL}$ water, mixed with $6 \mathrm{~g}$ of PVP powder, and refluxed for $3 \mathrm{~h}$. After cooling to room temperature, $20 \mathrm{~mL}$ of the silica solution was mixed with a $30 \mathrm{~mL} \mathrm{NaOH}$ solution (finally $0.04 \mathrm{~g} \mathrm{~mL}^{-1}$ of $\mathrm{NaOH}$ in $50 \mathrm{~mL}$ mixture) under continuous stirring. The resulting porous silica spheres were separated from the solution through centrifugation, washed 3 times with water, and finally dried at room temperature for $12 \mathrm{~h}$.

\section{Synthesis of porous $\mathrm{Fe}_{3} \mathrm{O}_{4} @ \mathrm{SiO}_{2}$ structures}

Superparamagnetic $\mathrm{Fe}_{3} \mathrm{O}_{4}$ particles with a diameter of $\sim 100$ $\mathrm{nm}$ were synthesized and then coated with $\mathrm{SiO}_{2}$ using a procedure developed previously in our group [30,31]. Then the product $\mathrm{Fe}_{3} \mathrm{O}_{4} @ \mathrm{SiO}_{2}$ was dispersed in a solution containing $20 \mathrm{~mL}$ of $\mathrm{H}_{2} \mathrm{O}$ and $1 \mathrm{~g}$ of PVP, followed by refluxing for $3 \mathrm{~h}$. Finally, the mixture was cooled down to which $5 \mathrm{~mL}$ of $\mathrm{NaOH}$ solution $\left(0.2 \mathrm{~g} \mathrm{~mL}^{-1}\right)$ was added to induce selective etching of the silica spheres. The final porous $\mathrm{Fe}_{3} \mathrm{O}_{4} @ \mathrm{SiO}_{2}$ structures were collected and washed with the procedure described above.

\section{Growth of CdSe within porous silica spheres}

The growth of CdSe nanocrystals in porous silica spheres was adapted from a literature procedure [31]. In a typical process, $25 \mathrm{mg}$ porous silica or $\mathrm{Fe}_{3} \mathrm{O}_{4} @ \mathrm{SiO}_{2}$ porous structures was added into a Se-TOP solution containing $0.158 \mathrm{~g}$ (2 mmol) of Se and $1.1 \mathrm{~mL}$ TOP $(2.4 \mathrm{mmol})$, and stirred at $90^{\circ} \mathrm{C}$ for about half an hour until the silica spheres dispersed well in the solution. In a separate flask, $\mathrm{CdO}$ $(0.0127 \mathrm{~g})$, stearic acid (0.114 g), and TOPO (1.94 g) were mixed in a $50 \mathrm{~mL}$ flask and heated to $150^{\circ} \mathrm{C}$ under vacuum. After adding $1 \mathrm{~mL}$ of oleylamine, the reaction system was heated to $320^{\circ} \mathrm{C}$ to form an optically clear solution under Ar protection, to which the porous silica-Se-TOP stock solution prepared above was injected. The temperature was then set at $290^{\circ} \mathrm{C}$ for the growth of QDs. At different time intervals, $1.5 \mathrm{~mL}$ reaction mixture was collected by a glass syringe and diluted by toluene for sampling. The QD-loaded porous silica spheres were separated from free CdSe nanocrystals by centrifugation at $6000 \mathrm{rpm}$ for $5 \mathrm{~min}$, and washed with toluene for 2 times.

\section{Measurement of quantum yield of porous $\mathrm{SiO}_{2} / \mathrm{CdSe}$ composite structures}

A standard dye Rhodamine 6G was chosen as a comparison to quantify the quantum yield of the porous $\mathrm{SiO}_{2} /$ CdSe composites [32,33]. At the excitation wavelength of $488 \mathrm{~nm}$, the photoluminescence (PL) spectra of the solutions of the composites and the dye were measured. The PL quantum yield of the composite samples were finally obtained by comparing the integrated PL intensities of the composites and the corresponding dye, assuming the quantum yield of the standard dye is $100 \%$.

\section{Estimation of CdSe loading number per sphere}

The number of CdSe incorporated in each porous silica sphere was estimated by the number ratio of CdSe nanocrystals to $\mathrm{SiO}_{2}$ beads in the solution. Briefly, the number of $\mathrm{SiO}_{2}$ was calculated by the amount of precursor TEOS, the density of normal colloidal $\mathrm{SiO}_{2}\left(2.2 \mathrm{~g} \mathrm{~cm}^{-3}\right)$, and the average size of $\mathrm{SiO}_{2}$ beads characterized by transmission electron microscope (TEM) images. On the other hand, the number of CdSe QDs was determined by the BeerLambert law. The size-dependent absorption coefficients of CdSe QDs were obtained from reference [34].

\section{Characterization}

The size and morphology of nanostructures were investigated using a Philips Tecnai 12 transmission electron microscope (TEM) operated at $120 \mathrm{keV}$. The composition and elemental distribution of the hollow structures were mapped through energy dispersive X-ray spectroscopy (EDS) by displaying the integrated intensity of each element as a function of the beam position. Both high-angle annular dark-field (HAADF) imaging and elemental mapping were recorded on a scanning transmission electron microscope (STEM, JEOL 2100F equipped with Oxford INCA energy dispersive spectrometer (EDS), Gatan's electron energy loss spectrometer (EELS) and Digiscan II STEM controller). UV-vis absorption measurements 
were carried out using a Varian Cary 50 spectrophotometer. During the measurements, all nanocrystal and dye solutions were placed in quartz cuvettes with a $1-\mathrm{cm}$ path length. The fluorescence emission spectra were measured by a Spex Flurolog Tau-3 fluorescence spectrophotometer with a xenon arc lamp source.

\section{RESULTS AND DISCUSSION}

In order to create mesoscale porosity, the silica spheres were etched by $\mathrm{NaOH}$ in the presence of PVP which served as a polymeric capping ligand that can bind to the silica surface [28]. After refluxing in distilled water for $3 \mathrm{~h}$, the surface of Stöber silica colloids was covered by PVP, which prevented a complete dissolution of the silica by $\mathrm{NaOH}$ in subsequent etching step, leaving behind a mesoporous silica framework. The porosity can be controlled by the extent of etching. The porous silica spheres were then transferred to a Se-TOP stock solution and formed a good dispersion. TOP molecules were found to play an important role in this modification, as they intensively coordinated with the oxygen from the silanol groups and rendered the silica particle hydrophobic characteristics and ensured particle dispersity in the solution $[35,36]$. Such coordination also took place on the mesopore framework, which helped to stabilize the Se precursors within the mesopores. Finally, the Se-loaded silica spheres were swiftly injected into the reaction flask containing cadmium precursor at high temperature $\left(\sim 320^{\circ} \mathrm{C}\right)$ to initiate the growth of CdSe. Samples taken out at different reaction time were washed by toluene, centrifuged to remove the free CdSe nanocrystals generated during the growth. Finally pure CdSe loaded silica spheres were collected and dispersed in toluene.

Fig. 1a shows a typical TEM image of the porous silica spheres with diameter about $450 \mathrm{~nm}$ after etching for $2 \mathrm{~h}$. The overall size did not decrease obviously during the etching process because of the PVP protection; while the rough surface and the low contrast of the spheres can be clearly observed, indicating the porous nature of the spheres. Following the incorporation of CdSe, the silica spheres collected by centrifugation became reddish, providing the first evidence of entrapment of CdSe QDs. As shown in Fig. 1b, regular bright field TEM image of the sample cannot clearly reveal the presence of CdSe QDs, although the contrast of the spheres seemingly increases and the spheres especially near surface regions becomes denser after loading with CdSe QDs. A better evidence is provided by the HAADF image in Fig. 1c, which shows a small portion of a composite sphere [37]. As the contrast of HAADF imaging is highly sensitive to the atomic-number of the materials, one can clearly appreciate the uniform size of CdSe dots (bright spots) and their homogeneous distribution inside the porous silica matrix.

The composition and elemental distribution of the composite nanostructures were mapped through EDS by displaying the integrated signal intensity as a function of the beam position when operating the TEM in the scanning mode. Mapping of elements including $\mathrm{Si}, \mathrm{O}, \mathrm{Cd}, \mathrm{Se}$, and $\mathrm{P}$ against composite spheres indicated that $\mathrm{CdSe}$ nanocrystals and the surfactant TOPO were uniformly distributed in the silica matrix. As shown in Fig. 2a, the $\mathrm{SiO}_{2}$ sphere contains a hollow interior due to extensive surface-protected etching. The CdSe nanocrystals appears to distribute mainly in the $\mathrm{SiO}_{2}$ porous network, but not in the hollow region at the center. On the other hand, porous $\mathrm{SiO}_{2}$ spheres without hollow interior can be also produced with less etching, and the CdSe nanocrystals are found to be distributed evenly throughout the corresponding composite spheres, as shown in similar elemental mapping analyses included as the Supplementary information. Although the element $\mathrm{P}$ also displays a uniform distribution, careful inspection suggests that the distribution of $\mathrm{P}$ followed the signals of $\mathrm{Si}$ and $\mathrm{O}$. The integrated EDS spectrum of a single compos-
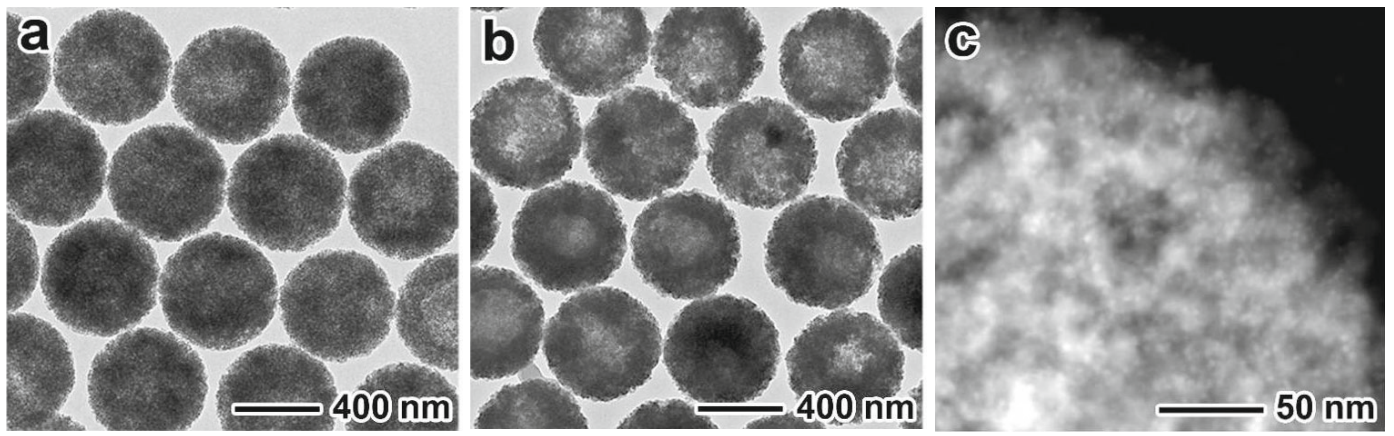

Figure 1 (a) TEM image of porous silica spheres produced by etching with $\mathrm{NaOH}$ with the protection of PVP. (b) TEM image of porous silica spheres containing CdSe nanocrystals. (c) High angle annular dark-field image of a portion of a composite sphere, showing uniformly distributed CdSe QDs (white dots) inside the porous silica matrix. 

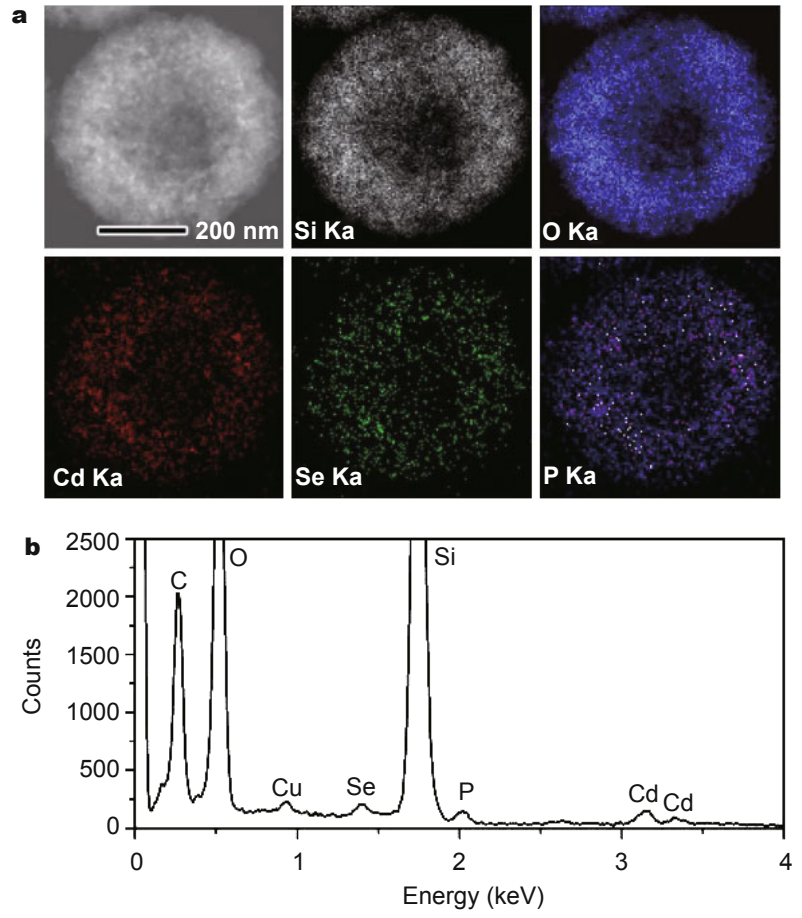

Figure 2 (a) Elemental mapping of $\mathrm{Si}, \mathrm{O}, \mathrm{Cd}, \mathrm{Se}$, and $\mathrm{P}$ against a typical $\mathrm{SiO}_{2} / \mathrm{CdSe}$ composite sphere through EDS, confirming uniform distribution of CdSe in the silica matrix. A hollow silica sphere is intentionally chosen to show that $\mathrm{CdSe}$ dots are trapped in the porous silica network, not the hollow interior. The first image is a conventional TEM image. (b) Integrated EDS spectrum of a single composite sphere. The strong signal of $\mathrm{C}$ and weak signal of $\mathrm{Cu}$ are originated from the supporting carbon film and the copper grid.

ite sphere displayed in Fig. 2b further clearly confirms the composition of $\mathrm{Si}, \mathrm{O}, \mathrm{Cd}, \mathrm{Se}$, and $\mathrm{P}$. The strong signal of $\mathrm{C}$ and weak signal of $\mathrm{Cu}$ were originated from the supporting carbon film and the copper grid from the TEM substrate, although it is believed that the surfactant TOPO might also contribute to the overall $\mathrm{C}$ signal. On the basis of above measurements, one can conclude that the CdSe nanocrystals have been uniformly loaded to the porous network of the $\mathrm{SiO}_{2}$ spheres.

We then characterized the optical properties of $\mathrm{SiO}_{2} /$ $\mathrm{CdSe}$ composite particles. A series of porous silica beads collected at different growth stages were shown in Fig. 3. The $\mathrm{SiO}_{2} / \mathrm{CdSe}$ composites in solution are highly luminescent (Fig. 3a). The quantum yield of the red sample could reach $40 \%$ compared with standard dye of rhodamine $6 \mathrm{G}$. This remarkable brightness here can be attributed to the large number CdSe incorporated and extra protection provided by silica walls. PL and UV-vis absorption spectra of the samples corresponding to various growth time of 8,15 , 45 and 120 s are shown in Fig. 3b, with emitting peaks at
530, 550, 580 and $595 \mathrm{~nm}$. True color fluorescence images of silica beads containing different single-color CdSe are also shown in Figs $3 \mathrm{c}-\mathrm{f}$, revealing that the beads are significantly brighter than the constituting single QDs, which proves the high loading amount of CdSe nanoparticles again.

After separating the mixture using centrifugation, two parts of the product were obtained: the free CdSe QDs dispersed in the supernatant and the $\mathrm{SiO}_{2} / \mathrm{CdSe}$ composite spheres as precipitates. Interestingly, the fluorescent colors of these two parts were different under UV light and the corresponding PL spectra further proved the shifts of the PL peak positions. As shown in Fig. 4, the solid curves stand for the free CdSe QDs, and the broken curves represent $\mathrm{SiO}_{2} / \mathrm{CdSe}$ composite structures. Very obvious blue shift was observed for all the composite spheres, indicating that the particle size of CdSe within the porous silica was smaller than the outside one. This difference becomes smaller with the time prolonged: the $\mathrm{SiO}_{2} / \mathrm{CdSe}$ composites with green color has the largest shift, and the red ones shifts very slightly (Figs $4 \mathrm{a}-\mathrm{d}$ ). It demonstrates that the confinement of the porous silica structures to the growth of CdSe does exist, most likely due to the limited diffusion of the growing species to the pores. As reaction time prolonged, such a difference becomes smaller because the growth of the particles outside slows down dramatically due to the decrease in specific surface energy, while those inside can catch up. On the other hand, the PL spectra of the composite structures have no obvious broadening in contrast to the free CdSe, indicating that the porous silica structure acts as a matrix to spatially separate the embedded CdSe nanocrystals.

The degree of porosity could be easily controlled by tuning the etching time, and the transmittance spectra were used to monitor the etching process [29]. We collected two kinds of porous silica spheres at different etching time, with transmittance at $1000 \mathrm{~nm}$ of $\sim 50 \%$ and $~ 80 \%$ in the spectrum, as shown in Fig. 5a. For convenience, these two different porous silica spheres are named as PSS1 and PSS2, respectively. The degree of etching apparent in the optical transmission measurements is fully consistent with the TEM characterization. Fig. 5b shows TEM images of these two corresponding silica spheres. When the transmittance is about $50 \%$, the silica spheres become porous, with an obvious rough surface. After the etching time was prolonged and transmittance reached $80 \%$, silica spheres with higher porosity PSS2 were obtained. Some of them have very low contrast in the center, indicating their hollow structures. Fig. 5c compares the loading abilities of PSS1 and PSS2 to CdSe nanocrystals. About $600 \mathrm{CdSe}$ nanocrystals are doped 

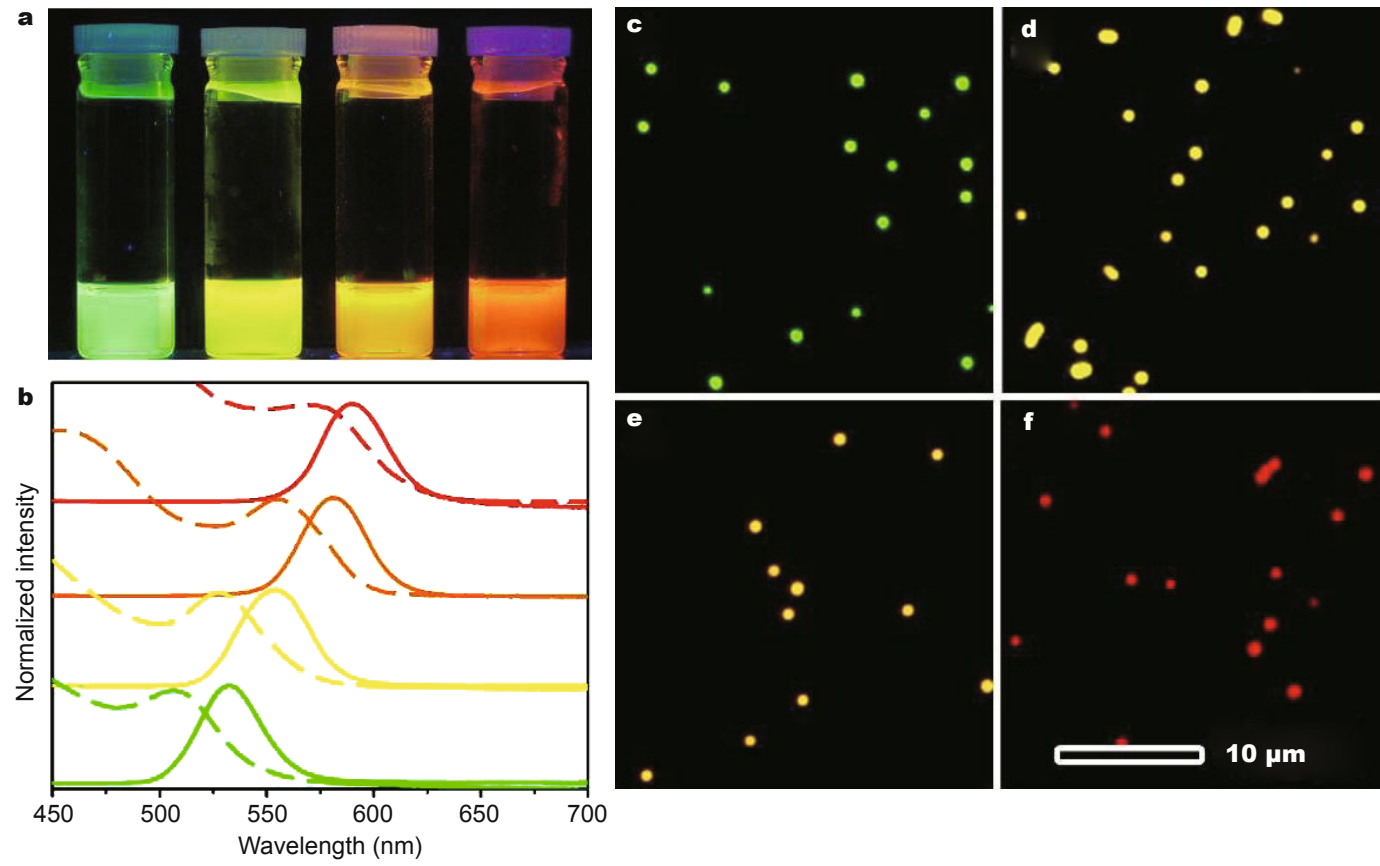

Figure 3 (a) Digital photo of a dispersion containing fluorescent $\mathrm{SiO}_{2} / \mathrm{CdSe}$ composite particles under the illumination of UV light with a wavelength of $\sim 365 \mathrm{~nm}$. All the samples were collected from the same reaction system but at different growth stages: from left to right, $8,15,45$ and $120 \mathrm{~s}$ after initiation of the reaction. The solutions only contain $\mathrm{SiO}_{2} / \mathrm{CdSe}$ composite particles as free CdSe nanocrystals have been removed by centrifugation. (b) Absorption and emission spectra of the composite particles collected at 8, 15, 45, and $120 \mathrm{~s}$ (from bottom to top). (c-f) Fluorescent micrographs of the composite particles in (b) dispersed on a glass cover slip.
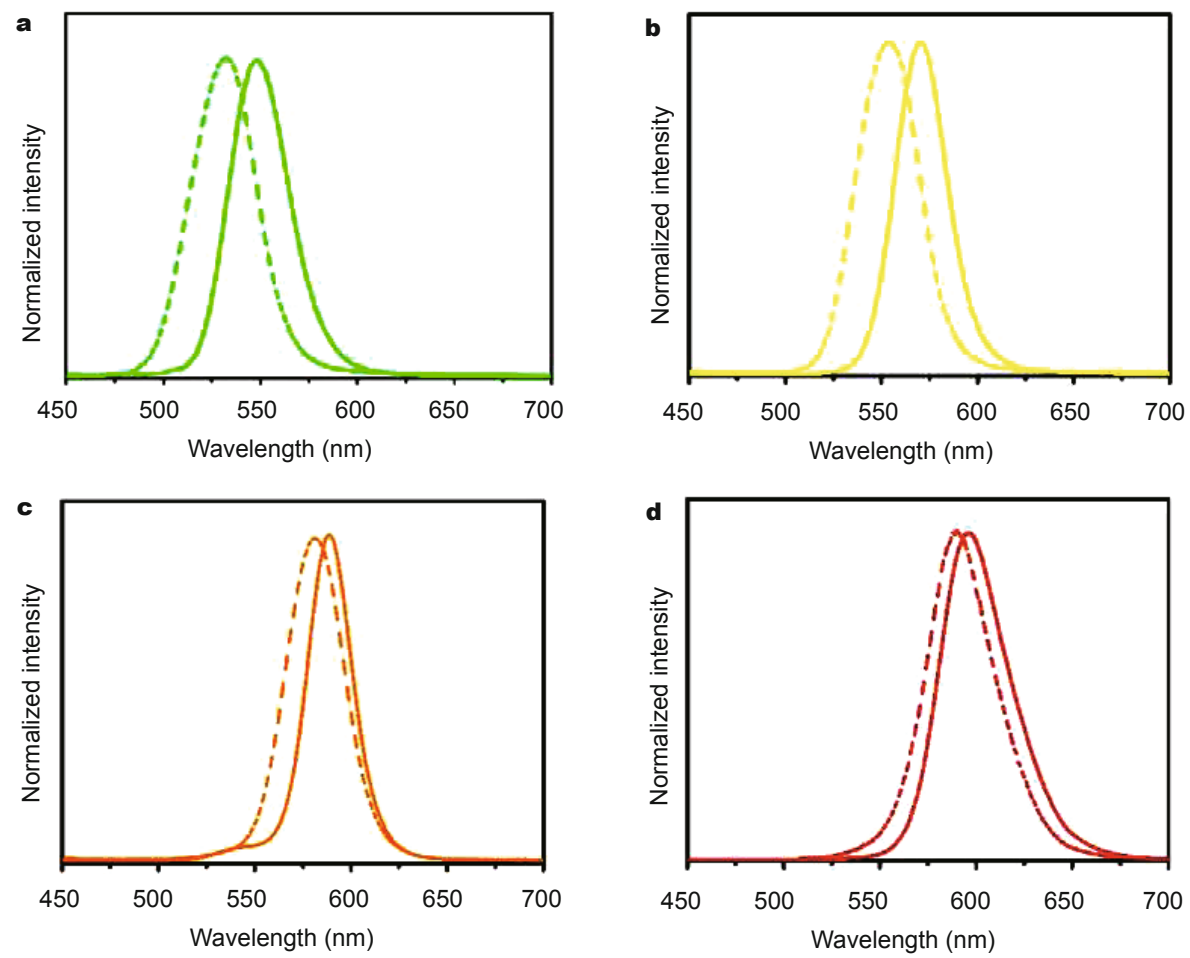

Figure 4 The confinement of porous silica to the growth of CdSe: PL spectra of the products collected at (a) 8, (b) 15, (c) 45, and (d) $120 \mathrm{~s}$ after initiation of the reaction. (The solid curves stand for the free CdSe QDs, and the broken curves were $\mathrm{CdSe} / \mathrm{SiO}_{2}$ spheres.) 

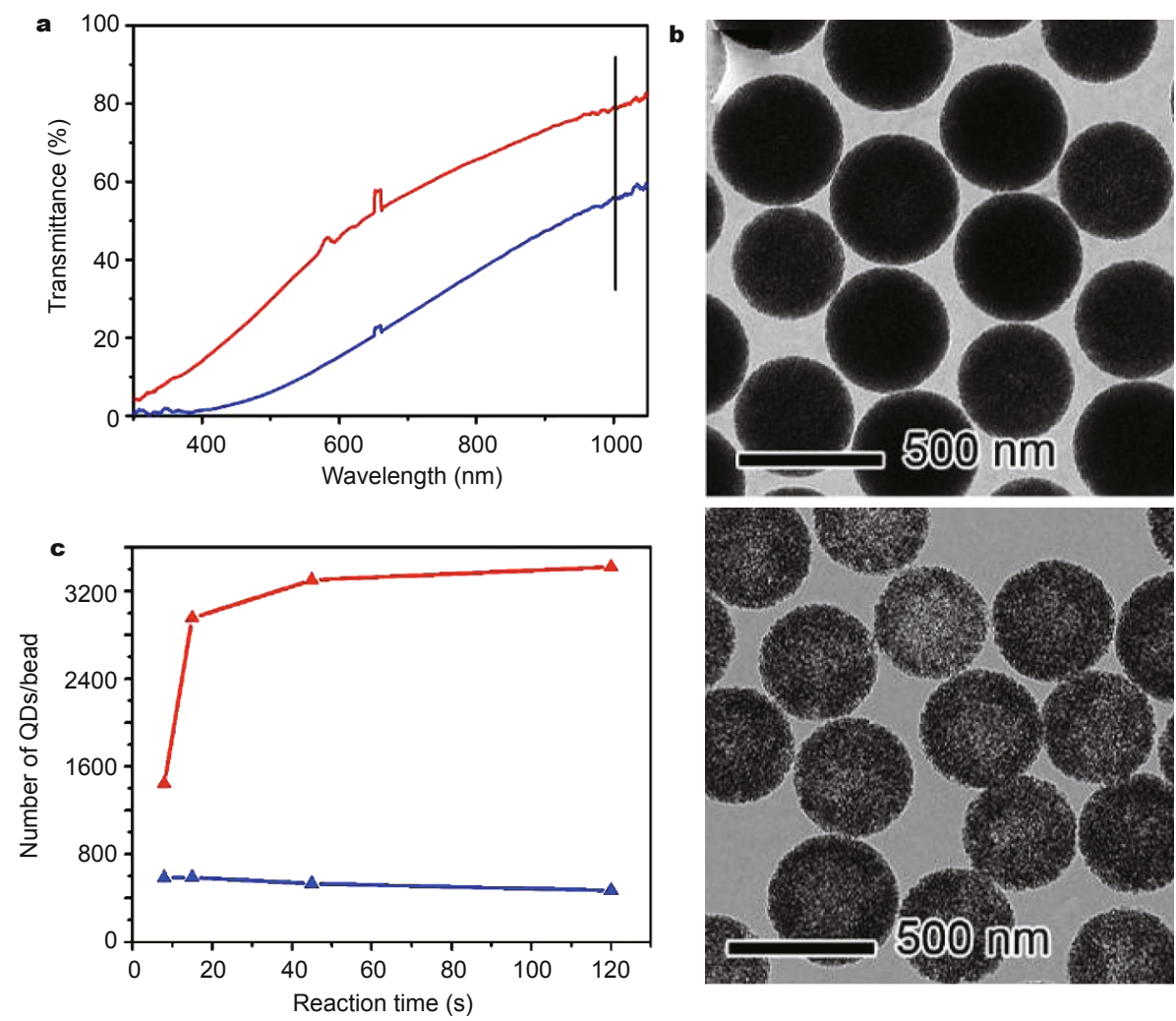

Figure 5 (a) The transmittance and (b) corresponding TEM images of different porous silica spheres that have been etched for different time, with higher transmittance and more porous structures at longer etching time. The blue line is with $~ 50 \%$ of transmittance at wavelength of $1000 \mathrm{~nm}$, and red line with $80 \%$ of transmittance. (c) The loading number of CdSe nanoparticles estimated by the optical absorption of the composites with different degrees of porosity.

in PSS1 in contrast to several thousands of the nanocrystals in the PSS2. Obviously, PSS2 can entrap more CdSe nanocrystals due to the more porous structures caused by longer etching time. Interestingly, during the growth process, the number of CdSe in PSS1 nearly kept the same. Two reasons may explain this phenomenon. First, the pores in PSS1 are relatively close to the sphere surface, which makes it not an issue for the diffusion of growing species. As a result, the number of CdSe in the silica spheres is considerably low and does not increase as the reaction prolonged. Second, the porous silica matrix prevents the CdSe seeds from aggregating with each other so that the number of CdSe QDs in PSS1 remains almost unchanged. In PSS2, the pores are not limited to the surface of the spheres, which allows more precursors to penetrate into the center of the spheres. However, the diffusion time of the growing species to the pores in the centre of PSS2 cannot be ignored in this case, as a result, in the first $60 \mathrm{~s}$ after injection, the number of CdSe nanocrystals continuously increased, from $\sim 1400$ to >3500 nanocrystals per PSS2 sphere. After that, the number of CdSe QDs kept the same and the size increased with the reaction time prolonged by consuming the growing species provided form the solution. The nearly unchanged number of CdSe at the later stage of growth again proved that the porous silica matrix kept the individual CdSe QDs well separated.

The size control of the porous $\mathrm{SiO}_{2} / \mathrm{CdSe}$ composites could be easily achieved by adjusting the initial size of the silica spheres; and the loading amount of QDs in each $\mathrm{SiO}_{2}$ sphere were related to the porosity degree of the sphere which can be tuned by varying etching times. Moreover, the ability to directly grow the nanoparticles in normal silica opens the door to the fabrication of a large variety of multifunctional composite nanostructures. Additional functional materials can now be incorporated as cores inside normal silica first, which can then form porous structures by surface-protected etching and entrap functional nanoparticles by the direct-growth method. Here, we have demonstrated this advantage by preparing $\mathrm{Fe}_{3} \mathrm{O}_{4} @$ $\mathrm{SiO}_{2} / \mathrm{CdSe}$ composite structures. First, superparamagnetic $\mathrm{Fe}_{3} \mathrm{O}_{4}$ particles with a diameter of $\sim 100 \mathrm{~nm}$ were prepared, followed by coating a layer of $\mathrm{SiO}_{2}$ with the thickness about 

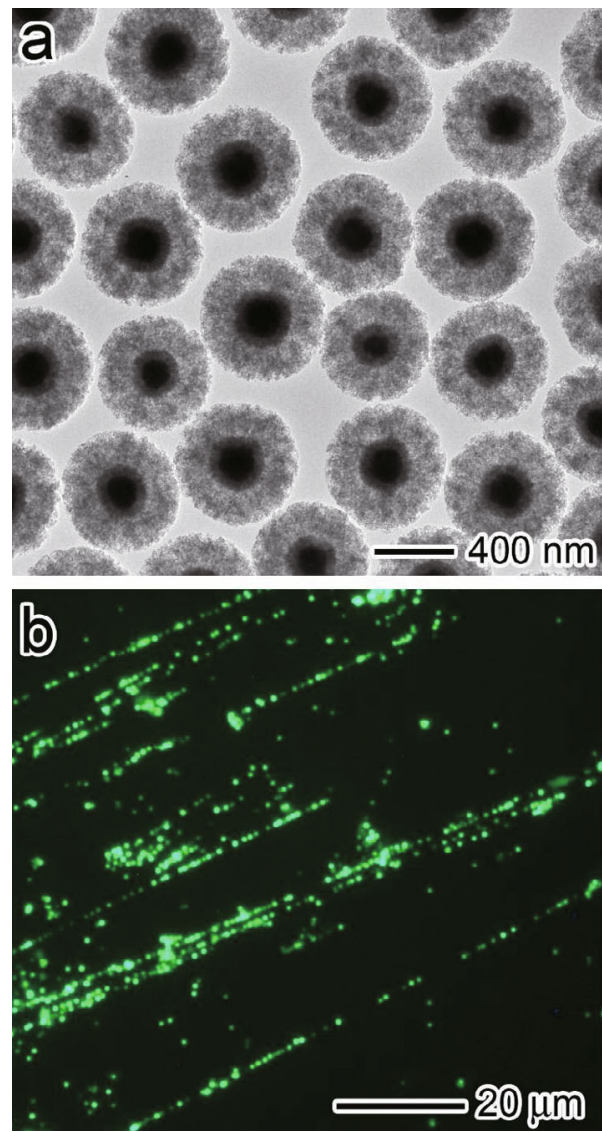

Figure 6 (a) TEM image of porous $\mathrm{Fe}_{3} \mathrm{O}_{4} @ \mathrm{SiO}_{2}$ core-shell composite particles containing $\mathrm{CdSe}$ nanocrystals. (b) Fluorescent micrograph showing linear structure of the $\mathrm{Fe}_{3} \mathrm{O}_{4} @ \mathrm{SiO}_{2} / \mathrm{CdSe}$ composite structures assembled under a magnetic field.

$180 \mathrm{~nm}$. After surface-protected etching, CdSe QDs were directly grown in the porous structures. Fig. 6a shows the TEM image of porous $\mathrm{Fe}_{3} \mathrm{O}_{4} @ \mathrm{SiO}_{2}$ core-shell structures containing $\mathrm{CdSe}$ nanocrystals. The dark area in the centre is the $\mathrm{Fe}_{3} \mathrm{O}_{4}$ core, and the outer layer with lower contrast is porous silica layer. Again, the CdSe particles cannot be easily identified in the silica matrix due to the similar contrast of silica domains. However, the strong fluorescent property of the composites proves the high loading amount of the CdSe QDs. Fig. 6b shows an optical microscopy image of the $\mathrm{Fe}_{3} \mathrm{O}_{4} @ \mathrm{SiO}_{2} / \mathrm{CdSe}$ core-shell composites when they were subjected to an external magnetic field and UV light irritation. The core-shell spheres can be self-assembled into linear chain structures, which demonstrate high magnetic sensitivity of the composite structures. Moreover, the observed strong green emission from the chain structures proves the high PL intensity. The combined magnetic-optical properties may endow the composites with many important technological applications.

\section{CONCLUSION}

In conclusion, we developed a new strategy for incorporating luminescent QDs to silica microspheres with controllable loading amounts. In comparison to embedding nanocrystals to conventional porous silica or polymer beads, this new system has several key features. First, the CdSe nanocrystals are directly nucleated and grown within the porous silica spheres so impregnation is not needed. Second, the pores have confinement effect on the growth of CdSe which leads to blue shift of PL spectra compared with the free ones. Third, the size of the silica spheres can be tuned by adjusting the original size of silica spheres, and as a result, uniform and size-tunable QD-loaded composites can be readily produced. Forth, by taking advantage of the easy control of porosity by etching time, the loading amount of CdSe nanocrystals can be conveniently adjusted. Fifth, functional materials can be incorporated as cores inside normal silica before loading QDs, which potentially opens a door to the fabrication of a large variety of multifunctional composite nanostructures.

Received 17 May 2015; accepted 4 June 2015; published online 16 June 2015

1 Murray CB, Norris DJ, Bawendi MG. Synthesis and characterization of nearly monodisperse CdE ( $\mathrm{E}=$ sulfur, selenium, tellurium) semiconductor nanocrystallites. J Am Chem Soc, 1993, 115: 8706-8715

2 Han M, Gao X, Su J, Nie S. Quantum-dot-tagged microbeads for multiplexed optical coding of biomolecules. Nat Biotechnol, 2001, 19: 631-635

3 Alivisatos AP. Semiconductor clusters, nanocrystals, and quantum dots. Science, 1996, 271: 933-937

4 Bruchez M, Moronne M, Gin P, Weiss S, Alivisatos AP. Semiconductor nanocrystals as fluorescent biological labels. Science, 1998, 281: 2013-2016

5 Murray CB, Kagan CR, Bawendi MG. Synthesis and characterization of monodispersenanocrystals and close-packed nanocrystalassemblies. Ann Rev Mater Sci, 2000, 30: 545-610

6 Chan WCW, Nie SM. Formation of high-quality CdTe, CdSe, and CdS nanocrystals using CdO as precursor. Science, 1998, 281: 2016-2018

7 Mattoussi H, Mauro JM, Goldman ER, et al. Self-assembly of CdSe$\mathrm{ZnS}$ quantum dot bioconjugates using an engineered recombinant protein. J Am Chem Soc, 2000, 122: 12142-12150

8 Selvan ST, Patra PK, Ang CY, Ying JY. Synthesis of silica-coated semiconductor and magnetic quantum dots and their use in the imaging of live cells. Angew Chem Int Ed, 2007, 46: 2448-2452

9 Selvan ST, Tan TT, Ying JY. Robust, non-cytotoxic, silica-coated CdSe quantum dots with efficient photoluminescence. Adv Mater, 2005, 17: 1620-1625

10 Nann T, Mulvaney P. Single quantum dots in spherical silica particles. Angew Chem Int Ed, 2004, 43: 5393-5396

11 Gao XH, Nie SM. Quantum dot-encoded mesoporous beads with high brightness and uniformity: rapid readout using flow cytometry. Anal Chem, 2004, 76: 2406-2410 
12 Song T, Zhang Q, Lu C, et al. Structural design and preparation of high-performance QD-encoded polymer beads for suspension arrays. J Mater Chem, 2011, 21: 2169-2177

13 Vaidya V, Couzis A, Maldarelli C. Reduction in aggregation and energy transfer of quantum dots incorporated in polystyrene beads by kinetic entrapment due to cross-linking during polymerization. Langmuir, 2015, 31: 3167-3179

14 Corato RD, Bigall NC, Ragusa A, et al. Multifunctional nanobeads based on quantum dots and magnetic nanoparticles: synthesis and cancer cell targeting and sorting. ACS Nano, 2011, 5: 1109-1121

15 Yang SJ, Nam S, Kim T, et al. Preparation and exceptional lithium anodic performance of porous carbon-coated $\mathrm{ZnO}$ quantum dots derived from a metal-organic framework. J Am Chem Soc, 2013, 135: 7394-7397

16 Liu H, Wu D, Liu Y, et al. Application of an optosensing chip based on molecularly imprinted polymer coated quantum dots for the highly selective and sensitive determination of sesamol in sesame oils. J Agric Food Chem, 2015, 63: 2545-2549

17 Chen PJ, Hu SH, Hung WH, Chen SY, Liu DM. Geometrical confinement of quantum dots in porous nanobeads with ultraefficient fluorescence for cell-specific targeting and bioimaging. J Mater Chem, 2012, 22: 9568-9575

18 Bao B, Li F, Li H, et al. pH-responsive dual fluorescent core-shell microspheres fabricated via a one-step emulsion polymerization. J Mater Chem C, 2013, 1: 3802-3807

19 Gao X, Nie S. Doping mesoporous materials with multicolor quantum dots. J Phys Chem B, 2003, 107: 11575-11578

20 Sathe TR, Agrawal A, Nie SM. Mesoporous silica beads embedded with semiconductor quantum dots and iron oxide nanocrystals: dual-function microcarriers for optical encoding and magnetic separation. Anal Chem, 2006, 78: 5627-5632

21 Gomez DE, Pastoriza-Santos I, Mulvaney P. Tunable whispering gallery mode emission from quantum-dot-doped microspheres. Small, 2005, 1: 238-241

22 Insin N, Tracy JB, Lee H, et al. Incorporation of iron oxide nanoparticles and quantum dots into silica microspheres. ACS Nano, 2008, 2: 197-202

23 Ki CD, Emrick T, Chang JY. Preparation of functional nanoporous silica for encapsulation of CdSe nanoparticles. Adv Mater, 2005, 17: 230-233

24 Mokari T, Sertchook H, Aharoni A, et al. Nano@micro: general method for entrapment of nanocrystals in sol-gel-derived composite hydrophobic silica spheres. Chem Mater, 2005, 17: 258-263

25 Zhelev Z, Ohba H, Bakalova R. Single quantum dot-micelles coated with silica shell as potentially non-cytotoxic fluorescent cell tracers. J Am Chem Soc, 2006, 128: 6324-6325

26 Ma Y, Li Y, Ma S, Bohn E. Highly bright water-soluble silica coated quantum dots with excellent stability. J Mater Chem B, 2014, 2: 5043-5051
27 Stober W, Fink A, Bohn E. Controlled growth of monodisperse silica spheres in the micron size range. J Colloid Interface Sci, 1968, 26: 62-69

28 Zhang Q, Zhang T, Ge J, Yin Y. Permeable silica shell through surface-protected etching. Nano Lett, 2008, 8: 2867-2871

29 Zhang Q, Ge J, Goebl J, et al. Rattle-type silica colloidal particles prepared by a surface-protected etching process. Nano Res, 2009, 2: 583-591

30 Ge J, Hu Y, Biasini M, Beyermann WP, Yin Y. Superparamagnetic magnetite colloidal nanocrystal clusters. Angew Chem Int Ed, 2007, 46: 4342-4345

31 Ge J, Yin Y. Magnetically tunable colloidal photonic structures in alkanol solutions. Adv Mater, 2008, 20: 3485-3491

32 Peng ZA, Peng X. Nearly monodisperse and shape-controlled CdSe nanocrystals via alternative routes: nucleation and growth. J Am Chem Soc, 2002, 124: 3343-3353

33 Grabolle M, Spieles M, Lesnyak V, et al. Determination of the fluorescence quantum yield of quantum dots: suitable procedures and achievable uncertainties. Anal Chem, 2009, 81: 6285-6294

34 Yu W, Qu L, Guo W, Peng X. Experimental determination of the extinction coefficient of CdTe, CdSe, and CdS nanocrystals. Chem Mater, 2003, 15: 2854-2860

35 Sommer J, Yang Y, Rambow D, Blumel J. Immobilization of phosphines on silica: identification of byproducts via ${ }^{31} \mathrm{P}$ CP/MAS studies of model alkyl-, aryl-, and ethoxyphosphonium salts. Inorg Chem, 2004, 43: 7561-7563

36 Blumel J. Reactions of phosphines with silicas: a solid-state NMR study. Inorg Chem, 1994, 33: 5050-5056

37 Max TO. High-angle annular dark-field imaging on a TEM/STEM system. J Electron Microsc Tech, 1991, 17: 221-230

Acknowledgements This work was supported by the US National Science Foundation (DMR-0956081). Li C acknowledges the fellowship support by the China Scholarship Council (CSC). We also thank the Central Facility for Advanced Microscopy and Microanalysis at University of California, Riverside, for help with TEM analysis. Work at the Molecular Foundry was supported by the Office of Science, Office of Basic Energy Sciences, of USA Department of Energy under Contract No. DE-AC02-05CH11231.

Author contributions Yin Y designed and supervised the project; Li C performed the experiments and wrote the paper; Lu Z, Zhang Q, Ge J and Shi $\mathrm{Z}$ contributed to the general discussion and reviewed the manuscript; Aloni S contributed to the TEM analysis.

Conflict of interest The authors declare that they have no conflict of interest.

Supplementary information Dark-field image and elemental mapping of the composite spheres are available in the online version of the paper. 


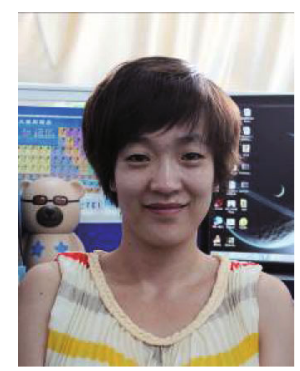

Chunguang Li received her PhD degree from the Department of Chemistry, Jilin University, Changchun, in 2010. Currently, she is an engineer at the State Key Laboratory of Inorganic Synthesis \& Preparative Chemistry, Jilin University. Her research interests focuses on the fabrication of colloidal inorganic nanostructures and their applications in biochemistry and photoelectronic fields.

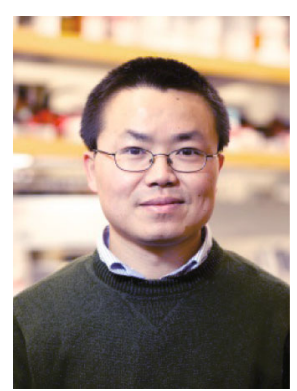

Yadong Yin received his PhD degree in materials science and engineering from the University of Washington in 2002, then worked as a postdoctoral fellow at the University of California, Berkeley and the Lawrence Berkeley National Laboratory (LBNL), and became a staff scientist at LBNL in 2005. In 2006 he joined the faculty at the Department of Chemistry at the University of California, Riverside. His research interests include the synthesis and application of nanostructured materials, self-assembly processes, and colloidal and interface chemistry.

中文摘要 本文报道了一种新颖且便捷的将荧光半导体纳米晶包覆到二氧化硅球中进而制备多功能纳米结构的方法. 从溶胶凝胶法合 成二氧化硅微球开始, 在聚合物配体保护下进行化学刻蚀从而获得介孔级孔道, 然后将带有孔道的二氧化硅微球投入到制备CdSe量子 点的高温反应体系中, 就可以直接在二氧化硅微球的孔道中生长尺寸均匀且具有高荧光产率的CdSe纳米晶. 研究发现二氧化硅微球中 的孔道对于 $\mathrm{CdSe}$ 量子点的生长有一定的抑制作用, 因此与自由生长的量子点比较其荧光光谱会发生蓝移, 二氧化硅微球中负载CdSe量 子点的数量可以通过选择不同刻蚀程度的二氧化硅微球来实现. 这种纳米结构优点在于: 不需要特殊的表面处理工艺, 对于不同形貌 及尺寸的二氧化硅微球具有普适性, 对于纳米晶与主体材料的尺寸调控具有较高的灵活性, 并且可以同时包裹其他功能材料, 比如将 磁性纳米粒子引入到该体系, 合成了兼具磁学与光学性能的 $\mathrm{Fe}_{3} \mathrm{O}_{4} @ \mathrm{SiO}_{2} / \mathrm{CdSe}$ 复合纳米结构材料. 该方法为制备多功能复合纳米结构 材料提供了一个平台. 